\title{
THE EQUIVALENCE OF THE LOG-SOBOLEV INEQUALITY AND A MIXING CONDITION FOR UNBOUNDED SPIN SYSTEMS ON THE LATTICE th
}

\author{
Nobuo YOSHIDA \\ Division of Mathematics, Graduate School of Science, Kyoto University, Kyoto 606-8502, Japan
}

Received in 20 June 1999, revised 20 June 2000

ABSTRACT. - We consider a ferromagnetic spin system with unbounded spin spaces on the $d$-dimensional integer lattice $(d \geqslant 1)$. We prove the equivalence of the log-Sobolev inequality, Poincaré inequality, and the exponential decay of the spin-spin correlation, which was originally obtained by D.W. Stroock and B. Zegarlinski [23,24] in the compact spin space setting. ( 2001 Éditions scientifiques et médicales Elsevier SAS

RÉSUMÉ. - Nous considérons un système de spins ferromagnétique avec un espace de spins non-borné, sur le réseau $\mathbf{Z}^{d}(d \geqslant 1)$. Nous montrons l'équivalence entre l'inégalité Sobolev logarithmique, l'inégalité de Poincaré et la décroissance exponentielle de la corrélation spinspin, qui fut montrée initialement par D.W. Stroock and B. Zegarlinski [23,24] dans le cas d'un espace de spins compact. @ 2001 Éditions scientifiques et médicales Elsevier SAS

\section{Introduction}

For lattice spin systems with the compact spin space, remarkable progresses have been made to understand the relation between the mixing properties of the Gibbs states and the speed at which the associated Glauber dynamics relaxes to equilibrium. In particular, the results obtained by D.W. Stroock and B. Zegarlinski [23,24] are very impressive. They state that a mixing property (the Dobrushin-Shlosman mixing condition) and the rapid relaxation property of the Glauber dynamics are, if properly defined, not only related to each other but in fact equivalent (see also works of F. Martinelli and E. Olivieri [20, 21]). The Dobrushin-Shlosman mixing condition referred to above is known to be true for example when the underlying lattice is one dimensional, or when the interaction potential is weak enough.

In this article, we consider a ferromagnetic lattice spin system with unbounded spin space. We prove in Theorem 2.1 below that the mixing conditions for the finite volume

\footnotetext{
${ }^{\text {W }}$ Last modified May 19, 1999; printed out today. The title in French: Equivalence entre l'inégalité Sobolev logarithmique et une condition de mélange pour les systèmes à espaces de spins non-borné sur le réseau.

E-mail address: nobuo@kusm.kyoto-u.ac.jp (N. Yoshida).
} 
Gibbs states as follows are equivalent (each statement being understood to be uniform in the volume and the boundary condition); (i): the uniform log-Sobolev inequality holds, (ii): the uniform Poincaré inequality holds, (iii): the spin-spin correlation decays exponentially. This equivalence can be seen as an extension of the results in $[23,24]$.

We begin by introducing the standard setup of the model.

The lattice: We will work on the $d$-dimensional integer lattice $\mathbf{Z}^{d}=\left\{x=\left(x^{i}\right)_{i=1}^{d}: x^{i} \in\right.$ $\mathbf{Z}$ ) on which we consider the $l_{\infty}$-metric; $d\left(x_{1}, x_{2}\right)=\max _{1 \leqslant i \leqslant d}\left|x_{1}^{i}-x_{2}^{i}\right|\left(x_{1}, x_{2} \in \mathbf{Z}^{d}\right)$. For a set $\Lambda \subset \mathbf{Z}^{d}$, diam $\Lambda$ and $|\Lambda|$ stand respectively for its diameter and the cardinality. We write $\Lambda \Subset \mathbf{Z}^{d}$ when $1 \leqslant|\Lambda|<\infty$. The distance between two subsets $\Lambda_{1}$ and $\Lambda_{2}$ of $\mathbf{Z}^{d}$ will be denoted by $d\left(\Lambda_{1}, \Lambda_{2}\right)$. For $R \geqslant 1$, the $R$-boundary of a set $\Lambda$ is defined by

$$
\partial_{R} \Lambda=\{x \notin \Lambda ; d(x, \Lambda) \leqslant R\} .
$$

The value of $R$ will eventually be chosen as the range $R(\mathbf{J})$ of the interaction we consider (see (1.7) below).

The configuration spaces: The configuration spaces are defined as follows:

$$
\begin{aligned}
\mathbf{R}^{\Lambda} & =\left\{\sigma=\left(\sigma_{x}\right)_{x \in \Lambda} ; \sigma_{x} \in \mathbf{R}\right\}, \quad \Lambda \subset \mathbf{Z}^{d}, \\
\Omega & =\mathbf{R}^{\mathbf{Z}^{d}} .
\end{aligned}
$$

The functions of the configuration: Function spaces $\mathcal{C}$ and $\mathcal{C}_{\Lambda}\left(\Lambda \subset \mathbf{Z}^{d}\right)$ on the configuration space $\Omega$ are introduced as follows:

$$
\mathcal{C}=\{f: \Omega \rightarrow \mathbf{R} \mid f \text { satisfies the properties }(\mathrm{C} 1) \text { and }(\mathrm{C} 2) \text { below }\} .
$$

(C1) There is $\Lambda \Subset \mathbf{Z}^{d}$ such that $f$ depends only on $\left(\sigma_{x}\right)_{x \in \Lambda}$ and is of $C^{1}$ with respect to these variables.

(C2)

$$
\|\| f\left\|\stackrel{\text { def. }}{=} \sum_{x \in \mathbf{Z}^{d}}\right\| \nabla_{x} f \|<\infty
$$

where

$$
\|f\|=\sup _{\sigma \in \Omega}|f(\sigma)|, \quad \nabla_{x} f(\sigma)=\frac{\partial}{\partial \sigma_{x}} f(\sigma) .
$$

For $f \in \mathcal{C}$, we denote by $S_{f}$ the minimal set among those $\Lambda$ 's which satisfy the property referred to in $(\mathrm{C} 1)$ above. We define

$$
\mathcal{C}_{\Lambda}=\left\{f \in \mathcal{C} ; S_{f} \subset \Lambda\right\}, \quad \Lambda \subset \mathbf{Z}^{d} .
$$

The Hamiltonian: For $\Lambda \Subset \mathbf{Z}^{d}$ and $\omega \in \Omega$, we define a function $H^{\Lambda, \omega}: \Omega \rightarrow \mathbf{R}$, by

$$
H^{\Lambda, \omega}(\sigma)=\frac{1}{2} \sum_{x, y \in \Lambda} J_{x, y}\left(\sigma_{x}-\sigma_{y}\right)^{2}+\sum_{x \in \Lambda}\left(U\left(\sigma_{x}\right)-\sum_{y \notin \Lambda} J_{x, y} \sigma_{x} \omega_{y}\right) .
$$

Here, the coupling constants $\mathbf{J}=\left(J_{x, y} \in \mathbf{R} ; x, y \in \mathbf{Z}^{d}\right)$ are such that 
N. YOSHIDA / Ann. Inst. H. Poincaré, Probabilités et Statistiques 37 (2001) 223-243

$$
\begin{gathered}
R(\mathbf{J}) \stackrel{\text { def. }}{=} \sup \left\{d(x, y) ; J_{x, y} \neq 0\right\}<\infty, \\
\|\mathbf{J}\| \stackrel{\text { def. }}{=} \sup _{x} \sum_{y}\left|J_{x, y}\right|<\infty, \\
J_{x, y}=J_{y, x} \geqslant 0 \quad \text { if } x \neq y .
\end{gathered}
$$

For the function $U: \mathbf{R} \rightarrow \mathbf{R}$ in (1.6), we consider the following conditions.

(U0) There exist $V, W \in C^{\infty}(\mathbf{R} \rightarrow \mathbf{R}), m \in(0, \infty)$ and $C_{1.12} \in(0, \infty)$ such that

$$
\begin{aligned}
U(s) & =V(s)+W(s) \quad \text { for all } s \in \mathbf{R}, \\
\inf _{s} V^{\prime \prime}(s) & \geqslant m, \\
\|W\|_{\infty}+\left\|W^{\prime}\right\|_{\infty} & \leqslant C_{1.12}
\end{aligned}
$$

where $\|W\|_{\infty}=\sup _{s}|W(s)|$.

(U1) For any $m>0$, there exist $V, W \in C^{\infty}(\mathbf{R} \rightarrow \mathbf{R})$ and $C_{1.12} \in(0, \infty)$ such that (1.10)-(1.12) hold.

A typical example of $U$ which satisfies (U1) is given by the following polynomial

$$
U(s)=\sum_{\nu=1}^{N} a_{2 v} s^{2 v}+a_{1} s
$$

where $N \geqslant 2, a_{1}, a_{2} \in \mathbf{R}, a_{4} \geqslant 0, \ldots, a_{2(N-1)} \geqslant 0$ and $a_{2 N}>0$. Since $a_{2}$ can be large negative value, $U$ in (1.13) may have arbitrarily deep double wells.

The finite volume Gibbs state: For $\Lambda \Subset \mathbf{Z}^{d}$ and a boundary condition $\omega \in \Omega$, we define a Borel probability measure $E^{\Lambda, \omega}$ on $\mathbf{R}^{\Lambda}$ by

$$
E^{\Lambda, \omega}\left(d \sigma_{\Lambda}\right)=\frac{\exp -H^{\Lambda, \omega}(\sigma)}{Z^{\Lambda, \omega}} \prod_{x \in \Lambda} d \sigma_{x}
$$

where $Z^{\Lambda, \omega}$ is the normalizing constant. The measure $E^{\Lambda, \omega}$ is called the finite volume Gibbs state.

The Vassershtein distance: We let $\mathcal{P}\left(\mathbf{R}^{\Lambda}\right)$ denote the set of Borel probability measures on $\mathbf{R}^{\Lambda}$. For a finite set $\Lambda$ and $\nu, \widetilde{v} \in \mathcal{P}\left(\mathbf{R}^{\Lambda}\right)$, we define

$$
\mathcal{R}^{\Lambda}(v, \widetilde{v})=\inf \left\{\sum_{x \in \Lambda} \int_{\mathbf{R}^{\Lambda} \times \mathbf{R}^{\Lambda}} \mu(d \sigma d \widetilde{\sigma})\left|\sigma_{x}-\widetilde{\sigma}_{x}\right| ; \mu \in \mathcal{K}(\nu, \widetilde{v})\right\},
$$

where

$$
\mathcal{K}(\nu, \widetilde{v})=\left\{\mu \in \mathcal{P}\left(\mathbf{R}^{\Lambda} \times \mathbf{R}^{\Lambda}\right) ; \mu\left(d \sigma \times \mathbf{R}^{\Lambda}\right)=v, \mu\left(\mathbf{R}^{\Lambda} \times d \widetilde{\sigma}\right)=\widetilde{v}\right\} .
$$

The function $(v, \widetilde{v}) \mapsto \mathcal{R}^{\Lambda}(v, \widetilde{v})$ is called the Vassershtein distance.

Expectation and covariance. In what follows, the following common notations for the expectation and the covariance with respect to a probability measure $m$ is used; for functions $f$ and $g$,

$$
m(f)=\int m(d \sigma) f(\sigma), \quad m(f ; g)=m(f g)-m(f) \cdot m(g) .
$$




\section{The result}

We are now ready to present main results of this paper. The first result suggests that "all happy families are alike" [11].

THEOREM 2.1. - Consider the following conditions.

(LS) There is $C_{2.1} \in(0, \infty)$ such that for all $\Lambda \Subset \mathbf{Z}^{d}, f \in \mathcal{C}_{\Lambda}$ and $\omega \in \Omega$

$$
E^{\Lambda, \omega}\left(f^{2} \log \frac{f^{2}}{E^{\Lambda, \omega}\left(f^{2}\right)}\right) \leqslant C_{2.1} E^{\Lambda, \omega}\left(\sum_{x \in \Lambda}\left|\nabla_{x} f\right|^{2}\right) .
$$

(SG) There is $C_{2.2} \in(0, \infty)$ such that for all $\Lambda \Subset \mathbf{Z}^{d}, f \in \mathcal{C}_{\Lambda}$ and $\omega \in \Omega$

$$
E^{\Lambda, \omega}(f ; f) \leqslant C_{2.2} E^{\Lambda, \omega}\left(\sum_{x \in \Lambda}\left|\nabla_{x} f\right|^{2}\right) .
$$

(DS1) There is $C_{2.3} \in(0, \infty)$ such that for all $\Lambda \Subset \mathbf{Z}^{d}, \omega \in \Omega$ and $f, g \in \mathcal{C}_{\Lambda}$,

$$
\left|E^{\Lambda, \omega}(f ; g)\right| \leqslant B_{2.3}(f, g) \exp \left(-d\left(S_{f}, S_{g}\right) / C_{2.3}\right),
$$

where the coefficient $B_{2.3}(f, g)$ depends only on ||$|f|||,|||g|||,\left|S_{f}\right|$ and $\left|S_{g}\right|$.

(DS2) There is $C_{2.4} \in(0, \infty)$ such that for all $\Lambda \Subset \mathbf{Z}^{d}, f \in \mathcal{C}_{\Lambda}, y \notin \Lambda$ and $\omega, \widetilde{\omega} \in \Omega$ with $\widetilde{\omega} \equiv \omega$ off $y$,

$$
\left|E^{\Lambda, \widetilde{\omega}}(f)-E^{\Lambda, \omega}(f)\right| \leqslant B_{2.4}(f)\left|\widetilde{\omega}_{y}-\omega_{y}\right| \exp \left(-d\left(S_{f}, y\right) / C_{2.4}\right),
$$

where the coefficient $B_{2.4}(f)$ depends only on ||$|f|||$ and $\left|S_{f}\right|$.

(DS3) There is $C_{2.5} \in(0, \infty)$ such that for all $\Lambda \Subset \mathbf{Z}^{d}, \omega \in \Omega$ and $x, y \in \Lambda$

$$
\left|E^{\Lambda, \omega}\left(\sigma_{x} ; \sigma_{y}\right)\right| \leqslant C_{2.5} \exp \left(-d(x, y) / C_{2.5}\right) .
$$

(DS4) There is $C_{2.6} \in(0, \infty)$ such that for all $\Lambda \Subset \mathbf{Z}^{d}, z \in \Lambda, y \notin \Lambda$ and $\omega, \widetilde{\omega} \in \Omega$ with $\widetilde{\omega} \equiv \omega$ off $y$,

$$
\left|E^{\Lambda, \widetilde{\omega}}\left(\sigma_{z}\right)-E^{\Lambda, \omega}\left(\sigma_{z}\right)\right| \leqslant C_{2.6}\left|\widetilde{\omega}_{y}-\omega_{y}\right| \exp \left(-d(z, y) / C_{2.6}\right),
$$

(CC) There exist $V \Subset \mathbf{Z}^{d}, K_{2.7} \in(0, \infty)$ and $\varepsilon_{2.7} \in(0,1)$ such that

$$
\begin{aligned}
K_{2.7}\left|\partial_{R} V\right| & \leqslant \varepsilon_{2.7}|V|, \\
\mathcal{R}^{\Lambda \cap(x+V)}\left(E^{\Lambda \cap(x+V), \omega}, E^{\Lambda \cap(x+V), \widetilde{\omega}}\right) & \leqslant K_{2.7} \sum_{y \in \partial_{R}(\Lambda \cap(x+V))}\left|\omega_{y}-\widetilde{\omega}_{y}\right|
\end{aligned}
$$

for all $\Lambda \Subset \mathbf{Z}^{d}, x \in \mathbf{Z}^{d}$ and $\omega, \widetilde{\omega} \in \Omega$.

The following hold:

(a) Suppose that condition (U0) is satisfied. Then conditions listed above are related as 


$$
\begin{aligned}
(\mathrm{LS}) \Rightarrow(\mathrm{SG}) \Rightarrow(\mathrm{DS} 1) & \Rightarrow(\mathrm{DS} 2) \\
\Downarrow & \Downarrow \\
& (\mathrm{DS} 3) \Rightarrow(\mathrm{DS} 4) \Leftrightarrow(\mathrm{CC}) .
\end{aligned}
$$

(b) Suppose that condition (U1) is satisfied. Then, (DS4) implies (LS), and therefore, conditions (LS), (SG), (DS1)-(DS4) and (CC) are equivalent.

Remark 2.1. - Equivalence of this kind was first proved in the compact spin setting by D. Stroock and B. Zegarlinski $[23,24]$. The discrete spin case in which the single spin space is a finite set was further investigated in several other papers, e.g., [19-21]. For unbounded spin case, B. Zegarlinski claims in [29, Theorem 5.1] that (DS3) implies (LS) for any $d \geqslant 1$ and in a certain restricted sense. Unfortunately, the proof of this result is not presented in [29]. After this paper was written, the author received an article [7] by T. Bodineau and B. Helffer, which contains a version of "(SG) $\Rightarrow$ (DS1)" (where $\Lambda$ is restricted to a class of fat boxes) shown by a different technique.

Remark 2.2. - In the compact spin setting, R.L. Dobrushin and S. Shlosman [10,11] introduced a mixing condition called "complete analyticity". Conditions (DS2)-(DS4) in Theorem 2.1 can be understood as the transposition of the complete analyticity in the unbounded spin setting: for example, condition (DS2) can be compared with condition (IIIc) in [11]. Condition (CC) is similar to the "constructive criterion" $C_{V}$ in [9]. Note however that, unlike in $C_{V},(2.8)$ is imposed for $\Lambda \cap(x+V)$ with arbitrary $\Lambda \Subset \mathbf{Z}^{d}$. It is easy to verify by the argument in [3, pp.. 202-203] or [27, pp. 38-39] that (CC) is satisfied if $\sup _{x} \sum_{y: y \neq x} J_{x, y}$ is sufficiently small.

Remark 2.3. - Sufficient conditions for the mixing conditions listed in Theorem 2.1 are studied in some recent papers:

- A direct application of the Bakry-Emery criterion [4] for (LS) is discussed in [2]. This is possible when $U$ is a convex function.

- In $d=1$, B. Zegarlinski proved (LS) without imposing any other assumptions than (U0) [29, Theorem 4.1]. In this case, the Bakry-Emery criterion is no longer available in general. Instead, one takes advantage of a stronger version of (DS3) which comes from the dimensionality.

- High temperature (i.e., $\sup _{x} \sum_{y: y \neq x} J_{x, y}$ is sufficiently small): Log-Sobolev inequality (LS) under this condition was studied first in [27] with the function $U$ satisfying assumption (U1) plus another technical condition. Then, alternative approaches were proposed by T. Bodineau, B. Helffer and M. Ledoux $[6,7,16]$, which prove (LS) at high temperature under assumption (U0). An observation made at the end of Remark 2.2 above proves (LS) at high temperature under condition (U1). I. Gentil and C. Roberto [13] investigate spectral gap (SG) at high temperature with functions $U$ which do not necessarily satisfy condition (U1).

Remark 2.4. - Conditions in Theorem 2.1 are stated uniformly over all $\Lambda \Subset \mathbf{Z}^{d}$. However, as is pointed out by F. Martinelli and E. Olivieri [20,21] in the finite spin space setting, it is sometime reasonable to restrict one's attention only to regular enough $\Lambda$ 's such as fat enough boxes, in order to avoid some pathological phenomena caused by $\Lambda$ 's with irregular shapes. In our case, it is clear from the proofs that Theorem 2.1 
remains true if each "for all $\Lambda \Subset \mathbf{Z}^{d}$ " is replaced by "for all $\Lambda \in \mathcal{B}\left(n_{0}\right)$ ", where $\mathcal{B}\left(n_{0}\right)$ is the set of generalized box with the minimal side-length $n_{0}$ defined in [27].

\section{Proof of Theorem 2.1}

\subsection{A lemma on integrability}

We begin by preparing integrability properties which are needed later.

LEMMA 3.1. - The following hold:

(a) If condition (U0) is satisfied, then there are $\varepsilon \in(0, \infty)$ and $C_{3.1} \in(0, \infty)$ such that

$$
E^{\Lambda, \omega} \exp \left(\varepsilon\left|\sigma_{z}-E^{\Lambda, \omega}\left(\sigma_{z}\right)\right|^{2}\right) \leqslant C_{3.1}
$$

for all $z \in \Lambda \Subset \mathbf{Z}^{d}$ and $\omega \in \Omega$.

(b) If condition (U1) is satisfied, then for any $n=1,2, \ldots$, there exists $C_{3.2}(n)=$ $C_{3.2}(U, \mathbf{J}, n) \in(0, \infty)$ such that

$$
E^{\Lambda, \omega}\left(f^{2} \log \frac{f^{2}}{E^{\Lambda, \omega}\left(f^{2}\right)}\right) \leqslant C_{3.2}\left(\left|S_{f}\right|\right) E^{\Lambda, \omega}\left(\sum_{x \in \Lambda}\left|\nabla_{x} f\right|^{2}\right) .
$$

for $\Lambda \Subset \mathbf{Z}^{d}, \omega \in \Omega$ and $f \in \mathcal{C}_{\Lambda}$.

Proof. - The proof of part (a), due to M. Sugiura [25], is presented in [28]. Part (b) follows from the proof of [27, Lemmas 3.1 and 3.2].

Remark 3.1. - It is known that (3.2) implies (3.1). See [1].

\subsection{Proof of (SG) $\Rightarrow$ (DS1)}

We prove that (SG) implies (DS1) by the "semi-group method" which is standard in the compact spin space setting, e.g., [17, Chapter I, Theorem 4.20] and [23, Lemmas 1.8 and 2.5 ]. Here, we follow the argument in [17]. We will also borrow techniques from [23], especially in the proof of Lemma 3.2 below.

We introduce now for the model we are considering, a random time evolution which is sometimes called the Glauber dynamics. Set

$$
\begin{aligned}
& \Theta=\left\{\theta=\left(\theta_{t, x}\right)_{t \geqslant 0, x \in \mathbf{Z}^{d}} \mid\left(\theta_{t, x}\right)_{t \geqslant 0} \in C([0, \infty) \rightarrow \mathbf{R})\right. \text { and } \\
& \left.\qquad \theta_{0, x}=0 \text { for all } x \in \mathbf{Z}^{d}\right\}, \\
& \mathbf{P}=\text { the Wiener measure on } \Theta .
\end{aligned}
$$

The second of these amounts to saying that $\left(\theta_{t, x}\right)_{t \geqslant 0}\left(x \in \mathbf{Z}^{d}\right)$ are independent standard Brownian motions under the probability measure P. For a set $\Lambda \Subset \mathbf{Z}^{d}$ and $\omega \in \Omega$, consider the following stochastic differential equation (SDE) for the unknown process 
$\sigma_{t}^{\Lambda, \omega}=\left(\sigma_{t, x}^{\Lambda, \omega}\right)_{x \in \mathbf{Z}^{d}}$

$$
\sigma_{t, x}^{\Lambda, \omega}= \begin{cases}\omega_{x}+\theta_{t, x}-\frac{1}{2} \int_{0}^{t} d s \nabla_{x} H^{\Lambda, \omega}\left(\sigma_{s}^{\Lambda, \omega}\right) & \text { if } x \in \Lambda, \\ \omega_{x} & \text { if } x \notin \Lambda .\end{cases}
$$

The existence and the uniqueness of the solution to (3.5) is well-known even for $\Lambda=\mathbf{Z}^{d}$ as well as for $\Lambda \Subset \mathbf{Z}^{d}$ (cf. [8]).

Our proof of (SG) $\Rightarrow$ (DS1) is based on the "finite speed propagation property" of the dynamics in the following form.

LEMMA 3.2. - For any $\varepsilon \in(0, \infty)$, there exists $C_{3.6} \in(0, \infty)$ such that

$$
\mathbf{P}\left(f\left(\sigma_{t}^{\Lambda, \omega}\right) ; g\left(\sigma_{t}^{\Lambda, \omega}\right)\right) \leqslant C_{3.6} \sum_{y, z \in \mathbf{Z}^{d}}\left\|\nabla_{y} f\right\|\left\|\nabla_{z} g\right\| \exp \left(C_{3.6} t-\varepsilon d(y, z)\right)
$$

for all $\Lambda \Subset \mathbf{Z}^{d}, \omega \in \Omega$ and $f, g \in \mathcal{C}_{\Lambda}$.

The proof of this lemma will be given in Section 3.3.

Remark 3.2. - In the compact spin setting, (3.6) is well known, e.g., [17, Chapter I, Proposition 4.18]. A different version of finite speed propagation property for the unbounded spin setting can be found in [29, Section 1].

Proof of $(\mathrm{SG}) \Rightarrow$ (DS1). - The proof is based on the idea as follows. If we have (SG), then, the distribution of the random variable $\sigma_{t}^{\Lambda, \omega}$ is close to $E^{\Lambda, \omega}$ for large, but finite time $t$. Suppose on the other hand that the supports $S_{f}$ and $S_{g}$ of functions $f, g \in \mathcal{C}_{\Lambda}$ are disjoint. Then, (3.6) says that the correlation of random variables $f\left(\sigma_{t}^{\Lambda, \omega}\right)$ and $g\left(\sigma_{t}^{\Lambda, \omega}\right)$ at any finite time $t$ are exponentially decaying in the distance of $S_{f}$ and $S_{g}$.

We now transpose the idea above into estimates. For $f \in \mathcal{C}_{\Lambda}$, set $\bar{f}(\sigma)=f(\sigma)-$ $E^{\Lambda, \omega} f$ and $\mathbf{P}_{t} f(\sigma)=\mathbf{P} f\left(\sigma^{\Lambda, \sigma_{\Lambda} \cdot \omega_{\Lambda^{c}}}\right)$. We then have that for $f, g \in \mathcal{C}_{\Lambda}$,

$$
E^{\Lambda, \omega}(f ; g)=J_{1}+J_{2}+J_{3},
$$

where

$$
\begin{aligned}
& J_{1}=E^{\Lambda, \omega}(\bar{f} \bar{g})-E^{\Lambda, \omega} \mathbf{P}_{t}(\bar{f} \bar{g}), \\
& J_{2}=E^{\Lambda, \omega} \mathbf{P}_{t}(\bar{f} \bar{g})-E^{\Lambda, \omega}\left(\mathbf{P}_{t} \bar{f} \cdot \mathbf{P}_{t} \bar{g}\right), \\
& J_{3}=E^{\Lambda, \omega}\left(\mathbf{P}_{t} \bar{f} \cdot \mathbf{P}_{t} \bar{g}\right) .
\end{aligned}
$$

We see from Lemma 3.2 that

$$
\left|J_{2}\right| \leqslant C_{3.6}|||f||||||g||| \exp \left(C_{3.6} t-\varepsilon d\left(S_{f}, S_{g}\right)\right) \text {. }
$$

By part (a) of Lemm 3.1, $E^{\Lambda, \omega}\left(\bar{f}^{2}\right)^{1 / 2} \leqslant E^{\Lambda, \omega}\left(\bar{f}^{4}\right)^{1 / 4} \leqslant\|\| f\|\|$. We therefore have by (SG) that 


$$
\begin{aligned}
\left|J_{1}\right| & \leqslant E^{\Lambda, \omega}\left|E^{\Lambda, \omega}(\bar{f} \bar{g})-\mathbf{P}_{t}(\bar{f} \bar{g})\right| \\
& \leqslant \exp \left(-t / C_{2.2}\right) E^{\Lambda, \omega}\left(\left|E^{\Lambda, \omega}(\bar{f} \bar{g})-\bar{f} \bar{g}\right|^{2}\right)^{1 / 2} \\
& \leqslant \exp \left(-t / C_{2.2}\right)\left(E^{\Lambda, \omega}\left(\bar{f}^{2}\right)^{1 / 2} E^{\Lambda, \omega}\left(\bar{g}^{2}\right)^{1 / 2}+E^{\Lambda, \omega}\left(\bar{f}^{4}\right)^{1 / 4} E^{\Lambda, \omega}\left(\bar{g}^{4}\right)^{1 / 4}\right) \\
& \leqslant 2 \exp \left(-t / C_{2.2}\right)|||f| \||||g|||, \\
\left|J_{3}\right| & \leqslant E^{\Lambda, \omega}\left(\left|\mathbf{P}_{t} \bar{g}\right|^{2}\right)^{1 / 2} E^{\Lambda, \omega}\left(\left|\mathbf{P}_{t} \bar{g}\right|^{2}\right)^{1 / 2} \\
& \leqslant \exp \left(-2 t / C_{2.2}\right) E^{\Lambda, \omega}\left(|\bar{f}|^{2}\right)^{1 / 2} E^{\Lambda, \omega}\left(|\bar{g}|^{2}\right)^{1 / 2} \\
& \leqslant \exp \left(-2 t / C_{2.2}\right)|||f| \||||g||| .
\end{aligned}
$$

Plugging (3.8), (3.9) and (3.10) into (3.7) and choosing $t=\varepsilon d\left(S_{f}, S_{g}\right) /\left(2 C_{3.6}\right)$, we obtain (DS1).

\subsection{Proof of Lemma 3.2}

LEMMA 3.3. - For $\varepsilon \in(0, \infty)$, define

$$
\begin{gathered}
l(\varepsilon)=\left\{\alpha=\left(\alpha_{x} \geqslant 0\right)_{x \in \mathbf{Z}^{d}} \mid\|\alpha\|_{\varepsilon} \stackrel{\text { def. }}{=} \sum_{x \in \mathbf{Z}^{d}} \alpha_{x} \exp (\varepsilon d(0, x))<\infty\right\}, \\
l(-\varepsilon)=\left\{\alpha=\left(\alpha_{x} \geqslant 0\right)_{x \in \mathbf{Z}^{d}} \mid\|\alpha\|_{-\varepsilon} \stackrel{\text { def. }}{=} \sum_{x \in \mathbf{Z}^{d}} \alpha_{x} \exp -\varepsilon d(0, x)<\infty\right\}, \\
\alpha * \beta=\left(\sum_{y \in \mathbf{Z}^{d}} \alpha_{x-y} \beta_{y}\right)_{x \in \mathbf{Z}^{d}}, \quad \text { for } \alpha \in l(\varepsilon) \text { and } \beta \in l(-\varepsilon) .
\end{gathered}
$$

Then the following hold: If $\alpha \in l(\varepsilon), \beta \in l(-\varepsilon)$ and $f_{t} \in l(-\varepsilon),(t \geqslant 0)$ are such that

$$
\begin{gathered}
\sup _{0 \leqslant s \leqslant t} \sum_{y \in \mathbf{Z}^{d}} f_{s, y} \exp -\varepsilon d(x, y)<\infty \\
f_{t, x} \leqslant \beta_{x}+\int_{0}^{t} d s\left(\alpha * f_{s}\right)_{x}
\end{gathered}
$$

for all $t \geqslant 0$ and $x \in \mathbf{Z}^{d}$. Then,

$$
f_{t, x} \leqslant \exp \left(t\|\alpha\|_{\varepsilon}\right) \sum_{y \in \mathbf{Z}^{d}}\left|\beta_{y}\right| \exp -\varepsilon d(x, y)
$$

for all $x \in \mathbf{Z}^{d}$ and $t \geqslant 0$.

Proof. - We begin by proving that for $\alpha \in l(\varepsilon), \beta \in l(-\varepsilon)$ and $x \in \mathbf{Z}^{d}$

$$
\left|\left((\alpha *)^{n} \beta\right)_{x}\right| \leqslant\|\alpha\|_{\varepsilon}^{n} \sum_{y \in \mathbf{Z}^{d}} \beta_{y} \exp -\varepsilon d(x, y), \quad n=1,2, \ldots,
$$

where $(\alpha *)^{n} \beta=\underbrace{\alpha * \cdots * \alpha *}_{n} \beta$. It is easy to prove that

$$
\left\|(\alpha *)^{n} \beta\right\|_{-\varepsilon} \leqslant\|\alpha\|_{\varepsilon}^{n}\|\beta\|_{-\varepsilon}, \quad n=1,2, \ldots
$$


We now introduce the shift transformation:

$$
\tau_{y}:\left(\alpha_{x}\right)_{x \in \mathbf{Z}^{d}} \mapsto\left(\alpha_{x+y}\right)_{x \in \mathbf{Z}^{d}}, \quad y \in \mathbf{Z}^{d} .
$$

Then, $\alpha *\left(\tau_{y} \beta\right)=\tau_{y}(\alpha * \beta)$ and hence $(\alpha *)^{n}\left(\tau_{y} \beta\right)=\tau_{y}\left((\alpha *)^{n} \beta\right)$. From this and (3.18), we see that

$$
\begin{aligned}
\left|\left((\alpha *)^{n} \beta\right)_{x}\right| & =\left|\left(\tau_{x}(\alpha *)^{n} \beta\right)_{0}\right| \\
& \leqslant\left\|\tau_{x}(\alpha *)^{n} \beta\right\|_{-\varepsilon} \\
& =\left\|(\alpha *)^{n} \tau_{x} \beta\right\|_{-\varepsilon} \\
& \leqslant\|\alpha\|_{\varepsilon}^{n}\left\|\tau_{x} \beta\right\|_{-\varepsilon},
\end{aligned}
$$

which implies (3.17).

To prove (3.16), we iterate (3.15) $(n-1)$-times to obtain that

$$
\begin{aligned}
f_{t, x} \leqslant & \sum_{m=0}^{n-1} \frac{t^{m}}{m !}\left((\alpha *)^{m} \beta\right)_{x}+\int_{0}^{t} d t_{n-1} \int_{0}^{t_{n-1}} d t_{n-2} \cdots \int_{0}^{t_{1}} d s\left((\alpha *)^{n} f_{s}\right)_{x} \\
\leqslant & \exp \left(t\|\alpha\|_{\varepsilon}\right) \sum_{y \in \mathbf{Z}^{d}}\left|\beta_{y}\right| \exp -\varepsilon d(x, y) \\
& +\left(t^{n}\|\alpha\|_{\varepsilon}^{n} / n !\right) \sup _{0 \leqslant s \leqslant t} \sum_{y \in \mathbf{Z}^{d}} f_{s, y} \exp -\varepsilon d(x, y),
\end{aligned}
$$

where we have used (3.17) to proceed to the second line. Since the second term on the right-hand side of (3.20) vanishes as $n \nearrow \infty$, we obtain (3.16).

Proof of Lemma 3.2. - We fix $\Lambda \Subset \mathbf{Z}^{d}$ and $\omega \in \mathbf{R}^{\Lambda^{c}}$ throughout this proof. Let us use the following notations; for $f \in C^{2}\left(\mathbf{R}^{\Lambda}\right)$,

$$
\begin{aligned}
\nabla_{x, y} f & =\nabla_{x} \nabla_{y} f, \\
A_{x} f & =\nabla_{x, x} f-\nabla_{x} H^{\Lambda, \omega} \nabla_{x} f, \\
A f & =\sum_{x \in \Lambda} A_{x} f .
\end{aligned}
$$

Step 1 .

$$
\frac{1}{2} A_{x}\left(\left|\nabla_{y} f\right|^{2}\right)-\nabla_{y} A_{x} f \cdot \nabla_{y} f \geqslant \begin{cases}-C_{3.24}\left|\nabla_{x} f \nabla_{y} f\right| & \text { if } d(x, y) \leqslant R, \\ 0 & \text { otherwise, }\end{cases}
$$

where $C_{3.24}=\sup _{x, y} J_{x, y}+\max \left\{0,-\inf _{s} U^{\prime \prime}(s)\right\}$. In fact, it is easy to see that the LHS of (3.24) is equal to

$$
\left|\nabla_{x, y} f\right|^{2}+\nabla_{x, y} H^{\Lambda, \omega} \nabla_{x} f \nabla_{y} f .
$$

Since $\nabla_{x, y} H^{\Lambda, \omega}=-J_{x, y}+\delta_{x, y} U^{\prime \prime}\left(\sigma_{x}\right),(3.25)$ is bounded from below by the RHS of (3.24).

Step 2. Take $f \in C_{\mathrm{c}}^{\infty}\left(\mathbf{R}^{\Lambda}\right)$ : the set of smooth functions on $\mathbf{R}^{\Lambda}$ which vanish outside a compact set and define $\mathbf{P}_{t} f(\sigma)=\mathbf{P} f\left(\sigma^{\Lambda, \sigma_{\Lambda} \cdot \omega_{\Lambda^{c}}}\right)$. Note that $\mathbf{P}_{t} f(\sigma)$ has an integral 
kernel $\mathbf{P}_{t}(\sigma, \tau)$ which is smooth in $(t, \sigma, \tau) \in(0, \infty) \times \mathbf{R}^{\Lambda} \times \mathbf{R}^{\Lambda}$ and solves $\frac{\partial}{\partial t} \mathbf{P}_{t}(\cdot, \tau)=$ $\frac{1}{2} A \mathbf{P}_{t}(\cdot, \tau)$. Therefore, $\mathbf{P}_{t} f(\sigma)$ is smooth in $(t, \sigma) \in(0, \infty) \times \mathbf{R}^{\Lambda}$ and solves $\frac{\partial}{\partial t} \mathbf{P}_{t} f=$ $\frac{1}{2} A \mathbf{P}_{t} f$. Let us prove that $\nabla_{x} \mathbf{P}_{t} f(\sigma)$ is bounded in $\sigma$ for any $x \in \Lambda$. This can be seen as follows. For a bounded smooth function $F=\left(F_{x}\right)_{x \in \Lambda}: \mathbf{R}^{\Lambda} \rightarrow \mathbf{R}^{\Lambda}$, define

$$
\begin{gathered}
\vec{A} F=\left(A F_{x}-\sum_{y \in \Lambda} \nabla_{x, y} H^{\Lambda, \omega} F_{y}\right)_{x \in \Lambda}, \\
\overrightarrow{\mathbf{P}}_{t} F(\sigma)=\mathbf{P}\left(M_{t} F\left(\sigma_{t}^{\Lambda, \sigma_{\Lambda} \cdot \omega_{\Lambda} c}\right)\right),
\end{gathered}
$$

where $M_{t}=\left(M_{t ; x, y}\right)_{x, y \in \Lambda}$ is a matrix defined as the solution to the following integral equation:

$$
M_{t ; x, y}=\delta_{x, y}-\frac{1}{2} \sum_{y \in \Lambda} \int_{0}^{t} d s M_{s ; x, z} \nabla_{z, y} H^{\Lambda, \omega}\left(\sigma_{s}^{\Lambda, \sigma_{\Lambda} \cdot \omega_{\Lambda} \mathrm{c}}\right) .
$$

We then have by standard arguments (see the proof of [26, Proposition 2.3] for example) that

- $\left(\overrightarrow{\mathbf{P}}_{t}\right)_{t \geqslant 0}$ extends to a strongly continuous semigroup on the Hilbert space $L^{2}\left(E^{\Lambda, \omega}\right) \otimes \mathbf{R}^{\Lambda}$

- $\vec{A}$ extends to the generator of the semigroup,

- $\left|M_{t} \xi\right| \leqslant|\xi| \exp \left(t C_{3.24} / 2\right)$, for all $\xi \in \mathbf{R}^{\Lambda}$ and $t \geqslant 0$, where $|\cdot|$ on each hand side stands for the Euclidean norm on $\mathbf{R}^{\Lambda}$.

These imply that

$$
\begin{aligned}
\frac{\partial}{\partial t} \overrightarrow{\mathbf{P}}_{t} F & =\frac{1}{2} \vec{A} \overrightarrow{\mathbf{P}}_{t} F=\frac{1}{2} \overrightarrow{\mathbf{P}}_{t} \vec{A} F, \\
\left|\overrightarrow{\mathbf{P}}_{t} F(\sigma)\right| & \leqslant \mathbf{P}_{t}(|F|)(\sigma) \exp \left(t C_{3.24} / 2\right) .
\end{aligned}
$$

On the other hand, it is easy to see that

$$
\nabla A f=\vec{A} \nabla f \quad \text { for } f \in C_{\mathrm{c}}^{\infty}\left(\mathbf{R}^{\Lambda}\right),
$$

where $\nabla f=\left(\nabla_{x} f\right)_{x \in \Lambda}$. It follows from (3.27), (3.28) and (3.29) that

$$
\left|\nabla \mathbf{P}_{t} f(\sigma)\right|=\left|\overrightarrow{\mathbf{P}}_{t} \nabla f(\sigma)\right| \leqslant \mathbf{P}_{t}(|\nabla f|)(\sigma) \exp \left(t C_{3.24} / 2\right),
$$

which implies that $\nabla_{x} \mathbf{P}_{t} f(\sigma)$ are bounded in $\sigma$ for any $x \in \Lambda$.

Step 3. Define $F_{t}=\left(F_{x, t}\right)_{x \in \mathbf{Z}^{d}}$ by

$$
F_{x, t}= \begin{cases}\left\|\nabla_{x} \mathbf{P}_{t} f\right\| & \text { if } x \in \Lambda, \\ 0 & \text { if } x \notin \Lambda .\end{cases}
$$

Let us prove that for any $\varepsilon \in(0, \infty)$, there is $C_{3.30} \in(0, \infty)$ such that

$$
F_{x, t} \leqslant C_{3.30} \sum_{y \in \mathbf{Z}^{d}} F_{y, 0} \exp \left(C_{3.30} t-2 \varepsilon d(x, y)\right) .
$$


To prove (3.30) by applying Lemma 3.3, we will check that

$$
\begin{gathered}
\sup _{0 \leqslant s \leqslant t} \sum_{y \in \mathbf{Z}^{d}} F_{y, s} \exp -2 \varepsilon d(x, y)<\infty, \\
F_{x, t} \leqslant F_{x, 0}+\int_{0}^{t} d s\left(\alpha * F_{s}\right)_{x}
\end{gathered}
$$

for all $t \geqslant 0$ and $x \in \mathbf{Z}^{d}$, where $\alpha_{x}=\frac{1}{2} C_{3.24} 1_{\{d(0, x) \leqslant R\}}$. (3.31) is obvious. To prove (3.32), define $\varphi(s)=\mathbf{P}_{t-s}\left(\left|\nabla_{x} \mathbf{P}_{s} f\right|^{2}\right), 0 \leqslant s \leqslant t$, for any fixed $t$. We then have by (3.24) that

$$
\begin{aligned}
\varphi^{\prime}(s) & =-\frac{1}{2} \mathbf{P}_{t-s} A\left(\left|\nabla_{x} \mathbf{P}_{s} f\right|^{2}\right)+2 \mathbf{P}_{t-s}\left(\nabla_{x} \mathbf{P}_{s} f \nabla_{x} \frac{A}{2} \mathbf{P}_{s} f\right) \\
& \leqslant C_{3.24} \sum_{y: d(x, y) \leqslant R} \mathbf{P}_{t-s}\left(\left|\nabla_{x} \mathbf{P}_{s} f\right|\left|\nabla_{y} \mathbf{P}_{s} f\right|\right) \\
& \leqslant C_{3.24} \sum_{y: d(x, y) \leqslant R} F_{x, s} F_{y, s} \\
& =2\left(\alpha * F_{s}\right)_{x} F_{x, s},
\end{aligned}
$$

from which we conclude

$$
\begin{aligned}
F_{x, t}^{2} & =\varphi(t) \leqslant \varphi(0)+2 \int_{0}^{t}\left(\alpha * F_{s}\right)_{x} F_{x, s} d s \\
& \leqslant F_{x, 0}^{2}+2 \int_{0}^{t}\left(\alpha * F_{s}\right)_{x} F_{x, s} d s .
\end{aligned}
$$

By solving (3.33) as an integral inequality with respect to $F_{x, t}$, we obtain (3.32).

Step 4. Take $f, g \in C_{\mathrm{c}}^{\infty}\left(\mathbf{R}^{\Lambda}\right)$ and define $G_{x, t}$ for $t \geqslant 0$ and $x \in \mathbf{Z}^{d}$ similarly as $F_{x, t}$ :

$$
G_{x, t}= \begin{cases}\left\|\nabla_{x} \mathbf{P}_{t} g\right\| & \text { if } x \in \Lambda, \\ 0 & \text { if } x \notin \Lambda .\end{cases}
$$

Let us prove that

$$
\sum_{x \in \mathbf{Z}^{d}} F_{x, t} G_{x, t} \leqslant C_{3.34} \sum_{y, z \in \mathbf{Z}^{d}}\left\|\nabla_{y} f\right\|\left\|\nabla_{z} f\right\| \exp \left(C_{3.34} t-\varepsilon d(y, z)\right),
$$

where $C_{3.34} \in(0, \infty)$ is independent of $\Lambda$ and $\omega$. Set $\gamma_{x}=\exp (-2 \varepsilon d(0, x))$. It is easy to see that for any $x \in \mathbf{Z}^{d}$,

$$
\exp (\varepsilon d(0, x)) \gamma_{x} \leqslant\|\gamma * \gamma\|_{\varepsilon} \leqslant\|\gamma\|_{\varepsilon}^{2} .
$$

Therefore, we have for any $y, z \in \mathbf{Z}^{d}$ that

$$
\sum_{x \in \mathbf{Z}^{d}} \gamma_{x-y} \gamma_{x-z}=\gamma * \gamma(y-z) \leqslant\|\gamma\|_{\varepsilon}^{2} \exp (-\varepsilon d(y, z)) .
$$


We now conclude (3.34) from (3.30) and (3.36) as follows:

$$
\begin{aligned}
\sum_{x \in \mathbf{Z}^{d}} F_{x, t} G_{x, t} & \leqslant C_{3.30}^{2} \exp \left(2 C_{3.30} t\right) \sum_{x \in \mathbf{Z}^{d}} \sum_{y, z \in \mathbf{Z}^{d}}\left\|\nabla_{y} f\right\|\left\|\nabla_{z} f\right\| \gamma_{x-y} \gamma_{x-z} \\
& \leqslant C_{3.30}^{2}\|\gamma\|_{\varepsilon}^{2} \exp \left(2 C_{3.30} t\right) \sum_{y, z \in \mathbf{Z}^{d}}\left\|\nabla_{y} f\right\|\left\|\nabla_{z} f\right\| \exp (-\varepsilon d(y, z)) .
\end{aligned}
$$

Step 5. Conclusion of the proof. For $f, g \in C_{\mathrm{c}}^{\infty}\left(\mathbf{R}^{\Lambda}\right)$, we define $\psi(s)=$ $\mathbf{P}_{s}\left(\mathbf{P}_{t-s} f \mathbf{P}_{t-s} g\right), 0 \leqslant s \leqslant t$ for any fixed $t$. We then have that

$$
\begin{aligned}
\psi^{\prime}(s) & =\mathbf{P}_{s}\left(\frac{1}{2} A\left(\mathbf{P}_{t-s} f \mathbf{P}_{t-s} g\right)-\frac{1}{2} A \mathbf{P}_{t-s} f \mathbf{P}_{t-s} g-\frac{1}{2} \mathbf{P}_{t-s} f A \mathbf{P}_{t-s} g\right) \\
& =\mathbf{P}_{s}\left(\sum_{x \in \Lambda} \nabla_{x} \mathbf{P}_{t-s} f \nabla_{x} \mathbf{P}_{t-s} g\right) \\
& \leqslant \sum_{x \in \Lambda} F_{x, t-s} G_{x, t-s},
\end{aligned}
$$

and therefore that

$$
\begin{aligned}
\mathbf{P}\left(f\left(\sigma_{t}^{\Lambda, \omega}\right) ; g\left(\sigma_{t}^{\Lambda, \omega}\right)\right) & =\mathbf{P}_{t}(f g)-\mathbf{P}_{t}(f) \mathbf{P}_{t}(g) \\
& =\psi(t)-\psi(0) \\
& \leqslant \int_{0}^{t} \sum_{x \in \Lambda} F_{x, t-s} G_{x, t-s} d s \\
& =\int_{0}^{t} \sum_{x \in \Lambda} F_{x, s} G_{x, s} d s .
\end{aligned}
$$

Plugging (3.34) into (3.37), we arrive at (3.6) for $f, g \in C_{\mathrm{c}}\left(\mathbf{R}^{\Lambda}\right)$. (3.6) for $f, g \in \mathcal{C}_{\Lambda}$ can be obtained by considering a suitable approximation.

\subsection{Proof of (DS4) $\Rightarrow(\mathrm{CC})$}

We will prove that

$$
\mathcal{R}^{\Lambda}\left(E^{\Lambda, \omega}, E^{\Lambda, \widetilde{\omega}}\right) \leqslant K_{3.38} \sum_{y \in \partial_{R} \Lambda}\left|\omega_{y}-\widetilde{\omega}_{y}\right|
$$

for all $\Lambda \Subset \mathbf{Z}^{d}$ and $\omega, \widetilde{\omega} \in \Omega$, where $K_{3.38}=C_{2.6} \sum_{z \in \mathbf{Z}^{d}} \exp \left(-|z| / C_{2.6}\right)$. Condition (CC) follows from (3.38) by taking $V$ as a large enough cube and applying (3.38) to $\Lambda \cap(x+V)$ instead of $\Lambda$. The proof of (3.38) reduces to the case $\omega \equiv \widetilde{\omega}$ off a site $y \in \partial_{R} \Lambda$. The reduction is a simple argument which uses the triangle inequality for the Vassershtein distance and an interpolating sequence $\omega_{0}, \ldots, \omega_{n}\left(n=\left|\partial_{R} \Lambda\right|\right)$ of boundary conditions such that $\omega_{0}=\omega, \omega_{n}=\widetilde{\omega}$ and $\omega_{j-1} \equiv \omega_{j}$ off a site in $\partial_{R} \Lambda(j=1, \ldots, n)$. 
We now assume that $\omega \equiv \widetilde{\omega}$ off a site $y \in \partial_{R} \Lambda$ and that $\omega_{y} \geqslant \widetilde{\omega}_{y}$ without loss. We then have by the FKG inequality that

$$
E^{\Lambda, \omega}\left(\bigcap_{x \in \Lambda}\left\{\sigma: \sigma_{x} \geqslant \tau_{x}\right\}\right) \geqslant E^{\Lambda, \widetilde{\omega}}\left(\bigcap_{x \in \Lambda}\left\{\sigma: \sigma_{x} \geqslant \tau_{x}\right\}\right)
$$

for all $\left(\tau_{x}\right)_{x \in \Lambda} \in \mathbf{R}^{\Lambda}$. From this and Strassen's theorem on the existence of an order preserving coupling $[18$, p. $129,(4.2)]$, there exists an measure $E^{\Lambda, \omega, \widetilde{\omega}} \in \mathcal{K}\left(E^{\Lambda, \omega}, E^{\Lambda, \widetilde{\omega}}\right)$ such that

$$
E^{\Lambda, \omega, \widetilde{\omega}}\left(\bigcap_{x \in \Lambda}\left\{(\sigma, \widetilde{\sigma}): \sigma_{x} \geqslant \widetilde{\sigma}_{x}\right\}\right)=1 .
$$

We have by (3.39) and (2.6) that

$$
\begin{aligned}
\mathcal{R}^{\Lambda}\left(E^{\Lambda, \omega}, E^{\Lambda, \widetilde{\omega}}\right) & \leqslant \sum_{z \in \Lambda} E^{\Lambda, \omega, \widetilde{\omega}}\left|\sigma_{z}-\widetilde{\sigma}_{z}\right| \\
& =\sum_{z \in \Lambda}\left(E^{\Lambda, \omega}\left(\sigma_{z}\right)-E^{\Lambda, \widetilde{\omega}}\left(\sigma_{z}\right)\right) \\
& \leqslant C_{2.6}\left|\omega_{y}-\widetilde{\omega}_{y}\right| \sum_{z \in \Lambda} \exp \left(-|z-y| / C_{2.6}\right) \\
& \leqslant K_{3.38}\left|\omega_{y}-\widetilde{\omega}_{y}\right|,
\end{aligned}
$$

which proves (3.38).

\subsection{Proof of $(\mathrm{CC}) \Rightarrow(\mathrm{DS} 4)$}

We follow the the argument in [27, pp. 33-38] which originally is adapted from [10, pp. 353-356]. We begin with lemmas.

LEMMA 3.4. - Suppose that $\Lambda \Subset \mathbf{Z}^{d}, y \notin \Lambda$ and $\omega, \widetilde{\omega} \in \Omega$ are such that $\omega \equiv \widetilde{\omega}$ off $y$. Then, there exists a measure $E^{\Lambda, \omega, \widetilde{\omega}} \in \mathcal{K}\left(E^{\Lambda, \omega}, E^{\Lambda, \widetilde{\omega}}\right)$ such that if we define $f_{z}^{\Lambda, \omega, \widetilde{\omega}}$ by

$$
f_{z}^{\Lambda, \omega, \widetilde{\omega}}=\int E^{\Lambda, \omega, \widetilde{\omega}}(d \sigma d \widetilde{\sigma})\left|\sigma_{z}-\widetilde{\sigma}_{z}\right|
$$

then

$$
\begin{aligned}
\sum_{z \in \Lambda} f_{z}^{\Lambda, \omega, \widetilde{\omega}} & =\mathcal{R}^{\Lambda}\left(E^{\Lambda, \omega}, E^{\Lambda, \widetilde{\omega}}\right), \\
\sup _{z \in \Lambda} f_{z}^{\Lambda, \omega, \widetilde{\omega}} & \leqslant C_{3.42}\left|\omega_{y}-\widetilde{\omega}_{y}\right|, \\
\sum_{z \in \Lambda \cap(x+V)} f_{z}^{\Lambda, \omega, \widetilde{\omega}} & \leqslant K_{2.7} \sum_{z \in \Lambda \cap\left(x+\partial_{R} V\right)} f_{z}^{\Lambda, \omega, \widetilde{\omega}}+K_{2.7}\left|\omega_{y}-\widetilde{\omega}_{y}\right| 1_{\{y \in x+V\}},
\end{aligned}
$$

where the constant $C_{3.42}$ depends only on $d, \mathbf{J}$ and $U$.

Proof. - We may assume $\omega_{y} \geqslant \widetilde{\omega}_{y}$ without loss for all $\left(\tau_{x}\right)_{x \in \Lambda} \in \mathbf{R}^{\Lambda}$. Therefore, there exists an measure $E^{\Lambda, \omega, \widetilde{\omega}} \in \mathcal{K}\left(E^{\Lambda, \omega}, E^{\Lambda, \widetilde{\omega}}\right)$ such that (3.39) holds. Therefore, if we define $f_{z}^{\Lambda, \omega, \widetilde{\omega}}$ by (3.40), we then have for any $z \in \Lambda$ and any $\mu \in \mathcal{K}\left(E^{\Lambda, \omega}, E^{\Lambda, \widetilde{\omega}}\right)$ that 


$$
\begin{aligned}
f_{z}^{\Lambda, \omega, \widetilde{\omega}} & =\int E^{\Lambda, \omega, \widetilde{\omega}}(d \sigma d \widetilde{\sigma})\left(\sigma_{z}-\widetilde{\sigma}_{z}\right) \\
& =E^{\Lambda, \omega}\left(\sigma_{z}\right)-E^{\Lambda, \widetilde{\omega}}\left(\sigma_{z}\right) \\
& =\int \mu(d \sigma d \widetilde{\sigma})\left(\sigma_{z}-\widetilde{\sigma}_{z}\right) \\
& \leqslant \int \mu(d \sigma d \widetilde{\sigma})\left|\sigma_{z}-\widetilde{\sigma}_{z}\right|,
\end{aligned}
$$

and hence that

$$
\sum_{z \in \Lambda} f_{z}^{\Lambda, \omega, \widetilde{\omega}} \leqslant \sum_{z \in \Lambda} \int \mu(d \sigma d \widetilde{\sigma})\left|\sigma_{z}-\widetilde{\sigma}_{z}\right|
$$

which implies (3.41).

We prove (3.42) as follows. We repeat the argument in the proof of (DS1) $\Rightarrow$ (DS2) to see that

$$
\begin{aligned}
f_{z}^{\Lambda, \omega, \widetilde{\omega}} & =E^{\Lambda, \omega}\left(\sigma_{z}\right)-E^{\Lambda, \widetilde{\omega}}\left(\sigma_{z}\right) \\
& \leqslant\left(\widetilde{\omega}_{y}-\omega_{y}\right) \sup _{\zeta \in \Omega} \sum_{x \in \Lambda} J_{x, y} E^{\Lambda, \zeta}\left(\sigma_{z} ; \sigma_{x}\right) .
\end{aligned}
$$

This estimate proves (3.42), since we have (3.1).

We now turn to the proof of (3.43). We begin by taking a measurable map $(\xi, \widetilde{\xi}) \mapsto$ $\widehat{E}^{x}(\cdot \mid \xi, \widetilde{\xi})$ from $\mathbf{R}^{\Lambda} \times \mathbf{R}^{\Lambda}$ to $\mathcal{P}\left(\mathbf{R}^{\Lambda \cap(x+V)} \times \mathbf{R}^{\Lambda \cap(x+V)}\right)$ such that

$$
\begin{aligned}
& \mathcal{R}^{\Lambda \cap(x+V)}\left(E^{\Lambda \cap(x+V), \xi}, E^{\Lambda \cap(x+V), \widetilde{\xi}}\right) \\
& =\int \widehat{E}^{x}(d \sigma d \widetilde{\sigma} \mid \xi, \widetilde{\xi}) \sum_{z \in \Lambda \cap(x+V)}\left|\sigma_{z}-\widetilde{\sigma}_{z}\right|,
\end{aligned}
$$

for all $(\xi, \widetilde{\xi})$. The possibility of this measurable selection can be shown as an application of [22, Thorem 12.1.10]. (Use also Lemma 12.1.7 in that book to check that the set of measures which attain $\mathcal{R}^{\Lambda \cap(x+V)}\left(E^{\Lambda \cap(x+V), \xi}, E^{\Lambda \cap(x+V), \widetilde{\xi}}\right)$ is measurable as a set-valued function of $(\xi, \widetilde{\xi})$.)

We now define a measure $\bar{E}^{x, \omega, \widetilde{\omega}} \in \mathcal{P}\left(\mathbf{R}^{\Lambda} \times \mathbf{R}^{\Lambda}\right)$ by

$$
\bar{E}^{x, \omega, \widetilde{\omega}}(A \times B)=\int_{A} E^{\Lambda, \omega, \widetilde{\omega}}(d \xi d \widetilde{\xi}) \int_{B} \widehat{E}^{x}(d \sigma d \widetilde{\sigma} \mid \xi, \widetilde{\xi}),
$$

where $A \subset \mathbf{R}^{\Lambda \backslash(x+V)} \times \mathbf{R}^{\Lambda \backslash(x+V)}$ and $B \subset \mathbf{R}^{\Lambda \cap(x+V)} \times \mathbf{R}^{\Lambda \cap(x+V)}$. It follows from the above definition that

$$
\begin{aligned}
& \bar{E}^{x, \omega, \widetilde{\omega}} \in \mathcal{K}\left(E^{\Lambda, \omega}, E^{\Lambda, \widetilde{\omega}}\right), \\
& \bar{E}^{x, \omega, \widetilde{\omega}}=E^{\Lambda, \omega, \widetilde{\omega}} \quad \text { on } \mathbf{R}^{\Lambda \backslash(x+V)} \times \mathbf{R}^{\Lambda \backslash(x+V)} .
\end{aligned}
$$

To see (3.43), it is sufficient to prove that

$$
\sum_{z \in \Lambda \cap(x+V)} f_{z}^{\Lambda, \omega, \widetilde{\omega}} \leqslant \sum_{z \in \Lambda \cap(x+V)} \bar{f}_{z}^{x, \omega, \widetilde{\omega}}
$$




$$
\sum_{z \in \Lambda \cap(x+V)} \bar{f}_{z}^{x, \omega, \widetilde{\omega}} \leqslant K_{2.7} \sum_{z \in \Lambda \cap\left(x+\partial_{R} V\right)} f_{z}^{\Lambda, \omega, \widetilde{\omega}}+K_{2.7}\left|\omega_{y}-\widetilde{\omega}_{y}\right| 1_{\{y \in x+V\}}
$$

where

$$
\bar{f}_{z}^{x, \omega, \widetilde{\omega}}=\int \bar{E}^{x, \omega, \widetilde{\omega}}(d \sigma d \widetilde{\sigma})\left|\sigma_{z}-\widetilde{\sigma}_{z}\right|
$$

The first inequality (3.48) can be seen as follows. Since (3.47) implies that $f_{z}^{\Lambda, \omega, \widetilde{\omega}}=$ $\bar{f}_{z}^{x, \omega, \widetilde{\omega}}$ for $z \notin W \cap(x+V)$, we have from this, (3.41) and (3.46) that

$$
\begin{aligned}
\sum_{z \in \Lambda \cap(x+V)}\left(f_{z}^{\Lambda, \omega, \widetilde{\omega}}-\bar{f}_{z}^{x, \omega, \widetilde{\omega}}\right) & =\sum_{z \in \Lambda}\left(f_{z}^{\Lambda, \omega, \widetilde{\omega}}-\bar{f}_{z}^{x, \omega, \widetilde{\omega}}\right) \\
& \leqslant \sum_{z \in \Lambda} f_{z}^{\Lambda, \omega, \widetilde{\omega}}-\mathcal{R}^{\Lambda}\left(E^{\Lambda, \omega}, E^{\Lambda, \widetilde{\omega}}\right) \\
& =0 .
\end{aligned}
$$

To prove the second inequality (3.49), we will use (3.45) and (2.8) as follows:

$$
\begin{aligned}
\sum_{z \in \Lambda \cap(x+V)} \bar{f}_{z}^{x, \omega, \widetilde{\omega}} & =\int E^{\Lambda, \omega, \widetilde{\omega}}(d \xi d \widetilde{\xi}) \int \widehat{E}^{x}(d \sigma d \widetilde{\sigma} \mid \xi, \widetilde{\xi}) \sum_{z \in \Lambda \cap(x+V)}\left|\sigma_{z}-\widetilde{\sigma}_{z}\right| \\
& \leqslant K_{2.7} \int E^{\Lambda, \omega, \widetilde{\omega}}(d \xi d \widetilde{\xi}) \sum_{z \in \partial_{R}(\Lambda \cap(x+V))}\left|\xi_{z}-\widetilde{\xi}_{z}\right| \\
& =K_{2.7} \sum_{z \Lambda \cap\left(x+\partial_{R} V\right)} f_{z}^{\Lambda, \omega, \widetilde{\omega}}+K_{2.7}\left|\omega_{y}-\widetilde{\omega}_{y}\right| 1_{\{y \in x+V\}} .
\end{aligned}
$$

This completes the proof of Lemma 3.4.

LEMMA 3.5. - For any $A \subset \Lambda, L \geqslant 1$ and $\omega, \widetilde{\omega} \in \Omega$ with $\omega \equiv \widetilde{\omega}$ off $y$,

$$
\begin{aligned}
& \sum_{z \in \Lambda} f_{z}^{\Lambda, \omega, \widetilde{\omega}} \exp \left(-d(z, A) / C_{3.51}\right) \\
& \leqslant B_{3.51} \sum_{\substack{z \in \Lambda \\
d(z, y) \leqslant L+D_{3.51}}} f_{z}^{\Lambda, \omega, \widetilde{\omega}} \exp \left(-d(z, A) / C_{3.51}\right) \\
& \quad+B_{3.51}\left|\omega_{y}-\widetilde{\omega}_{y}\right| \sum_{\substack{z ; d(z, y)>L \\
z+V \ni y}} \exp \left(-d(z, A) / C_{3.51}\right)
\end{aligned}
$$

where $f_{z}^{\Lambda, \omega, \widetilde{\omega}}$ is defined by (3.40), $D_{3.51}=\operatorname{diam}\left(V \cup \partial_{R} V\right), B_{3.51}=B_{3.51}\left(R, V, \varepsilon_{2.7}\right)$ and $C_{3.51}=C_{3.51}\left(R, V, \varepsilon_{2.7}\right)$.

Proof. - We choose $C_{3.51}=C_{3.51}\left(R, V, \varepsilon_{2.7}\right)$ so large that

$$
C_{3.52} \stackrel{\text { def. }}{=} \exp \left(-D_{3.51} / C_{3.51}\right)-\varepsilon_{2.7} \exp \left(D_{3.51} / C_{3.51}\right)>0 .
$$

We set

$$
e_{x}=\exp \left(-d(x, A) / C_{3.51}\right), \quad C_{3.53}=\exp \left(D_{3.51} / C_{3.51}\right) .
$$


We then define $l_{z}^{0}=\sum_{x: x+V \ni z} e_{x}, l_{z}^{1}=K_{2.7} \sum_{x: x+\partial_{R} V \ni z} e_{x}, l_{z}=l_{z}^{0}-l_{z}^{1}$ and $r_{z}=$ $\sum_{\substack{x: x+V \ni z \\ d(x, y) \leqslant L}} e_{x}$. Let us first prove that

$$
\sum_{z \in \Lambda} f_{z}^{\Lambda, \omega, \widetilde{\omega}} l_{z} \leqslant \sum_{z \in \Lambda} f_{z}^{\Lambda, \omega, \widetilde{\omega}} r_{z}+K_{2.7}\left|\omega_{y}-\widetilde{\omega}_{y}\right| \sum_{\substack{x: d(x, y) \geqslant L \\ x+V \ni y y}} e_{z} .
$$

We have by (3.43) that

$$
\begin{aligned}
\sum_{x: d(x, y)>L} e_{x} \sum_{z \in \Lambda \cap(x+V)} f_{z}^{\Lambda, \omega, \widetilde{\omega}} \leqslant & K_{2.7} \sum_{x: d(x, y)>L} e_{x} \sum_{\substack{z \in \Lambda \cap\left(x+\partial_{R} V\right)\\
}} f_{z}^{\Lambda, \omega, \widetilde{\omega}} \\
& +K_{2.7}\left|\omega_{y}-\widetilde{\omega}_{y}\right| \sum_{\substack{x: d(x, y) \geqslant L \\
x+V \ni y}} e_{z} .
\end{aligned}
$$

Since

$$
\begin{gathered}
\sum_{x: d(x, y)>L} e_{x} \sum_{z \in \Lambda \cap(x+V)} f_{z}^{\Lambda, \omega, \widetilde{\omega}}=\sum_{z \in \Lambda} f_{z}^{\Lambda, \omega, \widetilde{\omega}} \sum_{\substack{x: d(x, y)>L \\
x+V \ni z}} e_{x}=\sum_{z \in \Lambda} f_{z}^{\Lambda, \omega, \widetilde{\omega}}\left(l_{z}^{0}-r_{z}\right), \\
K_{2.7} \sum_{x: d(x, y)>L} e_{x} \sum_{z \in \Lambda \cap\left(x+\partial_{R} V\right)} f_{z}^{\Lambda, \omega, \widetilde{\omega}} \leqslant \sum_{z \in \Lambda} f_{z}^{\Lambda, \omega, \widetilde{\omega}} l_{z}^{1},
\end{gathered}
$$

it follows from (3.55) that

$$
\sum_{z \in \Lambda} f_{z}^{\Lambda, \omega, \widetilde{\omega}}\left(l_{z}^{0}-r_{z}\right) \leqslant \sum_{z \in \Lambda} f_{z}^{\Lambda, \omega, \widetilde{\omega}} l_{z}^{1}+K_{2.7}\left|\omega_{y}-\widetilde{\omega}_{y}\right| \sum_{\substack{x: d(x, y) \geqslant L \\ x+V \ni y}} e_{x},
$$

which is equivalent to (3.54).

Let us next prove that

$$
\begin{aligned}
& r_{z} \leqslant C_{3.56} e_{z}, \\
& r_{z}=0 \quad \text { if } d(z, y)>L+D_{3.51}, \\
& l_{z} \geqslant C_{3.58} e_{z},
\end{aligned}
$$

where $C_{3.56}, C_{3.58} \in(0, \infty)$ depend only on $R, V$ and $\varepsilon_{2.7}$. To verify (3.56) and (3.57), note first that an easy to prove fact that

$$
C_{3.53}^{-1} \leqslant e_{x} / e_{z} \leqslant C_{3.53} \quad \text { if } d(x, z) \leqslant D_{3.51}
$$

We thus see that

$$
r_{z} \leqslant C_{3.53} e_{z} \sum_{\substack{x: x+V \ni z \\ d(x, y) \leqslant L}} 1
$$

which proves (3.56) and (3.57).

On the other hand, it follows from (3.59) and (2.7) that 


$$
\begin{aligned}
l_{z} & \geqslant e_{z}\left\{C_{3.53}^{-1} \sum_{x: x+V \ni z} 1-K_{2.7} C_{3.53} \sum_{x: x+\partial_{R} V \ni z} 1\right\} \\
& \geqslant e_{z}\left\{C_{3.53}^{-1}|V|-C_{3.53} \varepsilon_{2.7}|V|\right\} \\
& =C_{3.52}|V| e_{z},
\end{aligned}
$$

which proves (3.58). By plugging (3.56), (3.57) and (3.58) into (3.54), we obtain (3.51).

\subsection{Proof of (DS4) $\Rightarrow$ (LS)}

To prove that (DS4) implies (LS), we will use the method of S.-L. Lu and H.-T. Yau [19], in which (SG) and (LS) in compact spin settings are obtained from a certain mixing condition. The following lemma plays a key role in carrying out the strategy of the above mentioned paper in the unbounded spin setting. The proof we present here is based mainly on that of [27, Lemma 3.4]. An idea in a recent paper [6] by T. Bodineau and B. Helffer is also used.

LEMMA 3.6. - Suppose that conditions (U0) and (DS4) are satisfied. Then, the following hold:

(a) There exists $C_{3.61} \in(0, \infty)$ such that

$$
\left|E^{\Lambda, \omega}\left(f ; \sigma_{x}\right)\right| \leqslant C_{3.61}|\Delta| \exp \left(-d(x, \Delta) / C_{2.6}\right) E^{\Lambda, \omega}(f ; f)^{1 / 2},
$$

whenever $f \in \mathcal{C}, \Lambda \Subset \mathbf{Z}^{d}, x \in \Lambda$ and $\Delta \subset \Lambda$ are such that $S_{f} \cap \Lambda \subset \Delta \subset \Lambda$.

(b) There exists $C_{3.62} \in(0, \infty)$ such that

$$
\begin{aligned}
& \left|E^{\Lambda, \omega}\left(f^{2} ; \sigma_{x}\right)\right| \\
& \leqslant C_{3.62}|\Delta| \exp \left(-d(x, \Delta) / C_{2.6}\right) \\
& \quad \times E^{\Lambda, \omega}\left(f^{2}\right)^{1 / 2}\left(E^{\Lambda, \omega}(f ; f)^{1 / 2}+E^{\Lambda, \omega}\left(f^{2} \log \frac{f^{2}}{E^{\Lambda, \omega}\left(f^{2}\right)}\right)^{1 / 2}\right),
\end{aligned}
$$

whenever $f \in \mathcal{C}, \Lambda \Subset \mathbf{Z}^{d}, x \in \Lambda$ and $\Delta \subset \Lambda$ are such that $S_{f} \cap \Lambda \subset \Delta \subset \Lambda$.

Proof. - Set $E^{\Lambda, \xi, \widetilde{\xi}}=E^{\Lambda, \xi} \otimes E^{\Lambda, \widetilde{\xi}} \in \mathcal{P}\left(\mathbf{R}^{\Lambda} \times \mathbf{R}^{\Lambda}\right)$. We have that

$$
\begin{aligned}
E^{\Lambda, \omega}\left(f ; \sigma_{x}\right) & =\frac{1}{2} \int E^{\Lambda, \omega, \omega}(d \sigma d \widetilde{\sigma})(f(\sigma)-f(\widetilde{\sigma}))\left(\sigma_{x}-\widetilde{\sigma}_{x}\right) \\
& =\frac{1}{2} \int E^{\Lambda, \omega, \omega}(d \xi d \widetilde{\xi})(f(\xi)-f(\widetilde{\xi})) \int E^{\Lambda \backslash \Delta, \xi, \widetilde{\xi}}(d \sigma d \widetilde{\sigma})\left(\sigma_{x}-\widetilde{\sigma}_{x}\right) \\
& =\frac{1}{2} \int E^{\Lambda, \omega, \omega}(d \xi d \widetilde{\xi})(f(\xi)-f(\widetilde{\xi}))\left(E^{\Lambda \backslash \Delta, \xi}\left(\sigma_{x}\right)-E^{\Lambda \backslash \Delta \widetilde{\xi}}\left(\sigma_{x}\right)\right),
\end{aligned}
$$

where the second equality can be verified by applying Markov property to $E^{\Lambda, \omega}(d \sigma)$ and $E^{\Lambda, \omega}(d \widetilde{\sigma})$ separatedly. Since $\xi \equiv \widetilde{\xi} \equiv \omega$ outside $\Lambda$ in (3.63), we see from (DS4) that

$$
\left|E^{\Lambda \backslash \Delta, \xi}\left(\sigma_{x}\right)-E^{\Lambda \backslash \Delta, \widetilde{\xi}}\left(\sigma_{x}\right)\right| \leqslant C_{2.6} \sum_{y \in \Delta}\left|\xi_{y}-\widetilde{\xi}_{y}\right| \exp \left(-d(x, \Delta) / C_{2.6}\right) .
$$


Plugging this into (3.63), we see that

$$
\left|E^{\Lambda, \omega}\left(f ; \sigma_{x}\right)\right| \leqslant C_{3.65} \exp \left(-d(x, \Delta) / C_{2.6}\right) \sum_{z \in \Delta} I_{1}(f, z),
$$

where

$$
I_{1}(f, z)=\int E^{\Lambda, \omega, \omega}(d \sigma d \widetilde{\sigma})|f(\sigma)-f(\widetilde{\sigma})|\left|\sigma_{z}-\widetilde{\sigma}_{z}\right| .
$$

We have by Schwarz inequality that

$$
\begin{aligned}
I_{1}(f, z) & \leqslant\left(\int E^{\Lambda, \omega, \omega}(d \sigma d \widetilde{\sigma})|f(\sigma)-f(\widetilde{\sigma})|^{2}\right)^{1 / 2}\left(\int E^{\Lambda, \omega, \omega}(d \sigma d \widetilde{\sigma})\left|\sigma_{z}-\widetilde{\sigma}_{z}\right|^{2}\right)^{1 / 2} \\
& =2 E^{\Lambda, \omega}(f ; f)^{1 / 2} E^{\Lambda, \omega}\left(\sigma_{z} ; \sigma_{z}\right)^{1 / 2} .
\end{aligned}
$$

Since it follows from (3.1) that $E^{\Lambda, \omega}\left(\sigma_{z} ; \sigma_{z}\right)$ is bounded from above by a constant $C$ which is independent of $\Lambda, \omega$ and $z$, we obtain

$$
\sum_{z \in \Delta} I_{1}(f, z) \leqslant 2 C^{1 / 2}|\Delta| E^{\Lambda, \omega}(f ; f)^{1 / 2} .
$$

This, in conjunction with (3.65), implies (3.61).

The proof of (3.62) is similar. We see from (3.65) that

$$
\left|E^{\Lambda, \omega}\left(f^{2} ; \sigma_{x}\right)\right| \leqslant C_{2.6} \exp \left(-d(x, \Delta) / C_{2.6}\right) \sum_{z \in \Delta} I_{1}\left(f^{2}, z\right) .
$$

We have by Schwarz inequality that

$$
I_{1}\left(f^{2}, z\right) \leqslant \sqrt{2} E^{\Lambda, \omega}(f ; f)^{1 / 2} I_{2}(f, z)^{1 / 2},
$$

where

$$
I_{2}(f, z)=\int E^{\Lambda, \omega, \omega}(d \sigma d \widetilde{\sigma})|f(\sigma)+f(\widetilde{\sigma})|^{2}\left|\sigma_{z}-\widetilde{\sigma}_{z}\right|^{2} .
$$

To estimate $I_{2}(z)$, we will use an inequality: $a b \leqslant \exp (a)+b \log b$ for $a, b \geqslant 0$ and the following fact which follows from (3.1)

$$
\sup _{\Lambda, \omega, z} \int E^{\Lambda, \omega, \omega}(d \sigma d \widetilde{\sigma}) \exp \left(\varepsilon\left|\sigma_{z}-\widetilde{\sigma}_{z}\right|^{2}\right)<\infty .
$$

We see that

$$
\begin{aligned}
I_{2}(f, z) & \leqslant 4 \int E^{\Lambda, \omega, \omega}(d \sigma d \widetilde{\sigma}) f(\sigma)^{2}\left|\sigma_{z}-\widetilde{\sigma}_{z}\right|^{2} \\
& =4 E^{\Lambda, \omega}\left(f^{2}\right) \int E^{\Lambda, \omega, \omega}(d \sigma d \widetilde{\sigma})\left|\sigma_{z}-\widetilde{\sigma}_{z}\right|^{2} \frac{f(\sigma)^{2}}{E^{\Lambda, \omega}\left(f^{2}\right)} \\
& \leqslant 4 E^{\Lambda, \omega}\left(f^{2}\right) \int E^{\Lambda, \omega, \omega}(d \sigma d \widetilde{\sigma}) \exp \left(\varepsilon\left|\sigma_{z}-\widetilde{\sigma}_{z}\right|^{2}\right)
\end{aligned}
$$




$$
\begin{gathered}
+(4 / \varepsilon) E^{\Lambda, \omega}\left(f^{2} \log \frac{f^{2}}{\varepsilon E^{\Lambda, \omega}\left(f^{2}\right)}\right) \\
\leqslant C_{3.72} E^{\Lambda, \omega}\left(f^{2}\right)+C_{3.72} E^{\Lambda, \omega}\left(f^{2} \log \frac{f^{2}}{E^{\Lambda, \omega}\left(f^{2}\right)}\right),
\end{gathered}
$$

where $C_{3.72} \in(0, \infty)$ is independent of $\Lambda, \omega, z$ and $f$. Putting (3.70) and (3.72) together, we obtain

$$
\begin{aligned}
& \sum_{z \in \Delta} I_{1}\left(f^{2}, z\right) \\
& \quad \leqslant \sqrt{2} E^{\Lambda, \omega}(f ; f)^{1 / 2} \sum_{z \in \Delta} I_{2}(f, z)^{1 / 2} \\
& \quad \leqslant \sqrt{2} C_{3.72}^{1 / 2}|\Delta| E^{\Lambda, \omega}(f ; f)^{1 / 2}\left(E^{\Lambda, \omega}\left(f^{2}\right)^{1 / 2}+E^{\Lambda, \omega}\left(f^{2} \log \frac{f^{2}}{E^{\Lambda, \omega}\left(f^{2}\right)}\right)^{1 / 2}\right) \\
& \leqslant \sqrt{2} C_{3.72}^{1 / 2}|\Delta| E^{\Lambda, \omega}\left(f^{2}\right)^{1 / 2}\left(E^{\Lambda, \omega}(f ; f)^{1 / 2}+E^{\Lambda, \omega}\left(f^{2} \log \frac{f^{2}}{E^{\Lambda, \omega}\left(f^{2}\right)}\right)^{1 / 2}\right)
\end{aligned}
$$

which, in conjunction with (3.69), implies (3.62).

Proof of $(\mathrm{DS} 4) \Rightarrow(\mathrm{LS})$. - The proof is divided into two steps:

$$
\begin{aligned}
(3.2) \text { and }(3.61) & \Rightarrow(\mathrm{SG}), \\
(3.2),(3.62) \text { and }(\mathrm{SG}) & \Rightarrow(L S) .
\end{aligned}
$$

Each of above steps can be carried out in the same way as in [27, Sections 4 and 5]. In the second step, (SG) is used to handle $E^{\Lambda, \omega}(f ; f)$ on the RHS of (3.62).

\subsection{Completion of the proof of Theorem 2.1}

$(\mathrm{LS}) \Rightarrow(\mathrm{SG})$; This is well known (cf. [12, Corollary 6.1.17]).

$(\mathrm{SG}) \Rightarrow(\mathrm{DS} 1)$; This has already been shown in Section 3.2.

$(\mathrm{DS} 1) \Rightarrow(\mathrm{DS} 2)$; Define $\zeta=\zeta(\theta) \in \Omega(0 \leqslant \theta \leqslant 1)$ by $\zeta_{x}=\theta \widetilde{\omega}_{x}+(1-\theta) \omega_{x}\left(x \in \mathbf{Z}^{d}\right)$, which interpolates two boundary conditions $\omega$ and $\widetilde{\omega}$. We then have that

$$
E^{\Lambda, \widetilde{\omega}}(f)-E^{\Lambda, \omega}(f)=\int_{0}^{1} d \theta \frac{\partial}{\partial \theta} E^{\Lambda, \zeta}(f) .
$$

On the other hand, we have by direct computations that

$$
\begin{aligned}
\frac{\partial}{\partial \theta} E^{\Lambda, \zeta}(f) & =-E^{\Lambda, \zeta}\left(f ; \frac{\partial H^{\Lambda, \zeta}}{\partial \theta}\right) \\
& =\left(\widetilde{\omega}_{y}-\omega_{y}\right) \sum_{x \in \Lambda} J_{x, y} E^{\Lambda, \zeta}\left(f ; \sigma_{x}\right) .
\end{aligned}
$$

We see from (3.76) and (DS1) that

$$
\left|\frac{\partial}{\partial \theta} E^{\Lambda, \zeta}(f)\right| \leqslant C_{3.77}\left|\widetilde{\omega}_{y}-\omega_{y}\right| \exp \left(-d\left(S_{f}, y\right) / C_{2.3}\right) .
$$


Putting (3.75) and (3.77) together, we get (DS2).

$(\mathrm{DS} 1) \Rightarrow(\mathrm{DS} 3)$; Trivial.

$(\mathrm{DS} 2) \Rightarrow(\mathrm{DS} 4)$; Trivial.

$(\mathrm{DS} 3) \Rightarrow(\mathrm{DS} 4)$; This can be done by putting $f(\sigma)=\sigma_{z}$ in the proof of (DS1) $\Rightarrow$ (DS2).

(DS4) $\Leftrightarrow(C C)$ : This has already been shown in Sections 3.4, 3.5.

$(\mathrm{DS} 4) \Rightarrow(\mathrm{LS})$ : This has already been shown in Section 3.6.

\section{Acknowledgement}

The author would like to thank T. Bodineau, B. Helffer and M. Ledoux for useful discussions and for communicating their works $[6,7,16]$ prior to publication. The author also would like to thank the anonymous referee for many valuable comments.

\section{REFERENCES}

[1] Aida S., Masuda T., Shigekawa I., Logarithmic Sobolev inequalities and exponential integrability, J. Funct. Anal. 126 (1) (1994) 83-101.

[2] Albeverio S., Kondratiev Yu.G., Röckner M., Dirichlet operators via stochastic analysis, J. Funct. Anal. 128 (1995) 102-138.

[3] Albeverio S., Kondratiev Yu.G., Röckner M., Tsikalenko T.V., Uniqueness of Gibbs states for quantum lattice systems, Probab. Theory Related Fields 108 (1997) 193-218.

[4] Bakry D, Emery M., Diffusions hypercontractives, in: Séminaire de Probabilités XIX, Springer Lecture Notes in Math., Vol. 1123, 1985, pp. 177-206.

[5] Bellissard J., Høegh-Krohn R., Compactness and maximal Gibbs state for random Gibbs fields on the lattice, Comm. Math. Phys. 84 (1982) 297-327.

[6] Bodineau T., Helffer B., Log-Sobolev inequality for unbounded spin systems, J. Funct. Anal. 166 (1999) 168-178.

[7] Bodineau T., Helffer B., Correlations, spectral gap and log-Sobolev inequality for unbounded spin systems, in: Differential Equations and Mathematical Physics, Birmingham, International Press, 1999, pp. 27-42.

[8] Doss H., Royer G., Processus de diffusion associe aux mesures de Gibbs, Z. Wahrsch. verw. Gebiete 46 (1978) 107-124.

[9] Dobrushin R.L., Shlosman S., Constructive criterion for the uniqueness of Gibbs field, in: Fritz J., Jaffe A., Szasz D. (Eds.), Statistical Physics and Dynamical Systems, Birkhäuser, 1985.

[10] Dobrushin R.L., Shlosman S., Completely analytical Gibbs fields, in: Fritz J., Jaffe A., Szasz D. (Eds.), Statistical Physics and Dynamical Systems, Birkhäuser, 1985.

[11] Dobrushin R.L., Shlosman S., Completely analytical interactions: Constructive description, J. Stat. Phys. 46 (1987) 983-1014.

[12] Deuschel J.D., Stroock D.W., Large Deviations, Academic Press, 1989.

[13] Gentil I., Roberto C., Spectral gaps for spin systems: some non-convex phase examples, preprint, 2000.

[14] Helffer B., Remarks on the decay of correlations and Witten Laplacians III - Application to logarithmic Sobolev inequalities, Ann. de l'Insti. H. Poincaré (Sect. Probab-Stat) (1998) to appear.

[15] Holley R., Stroock D.W., Logarithmic Sobolev inequality and stochastic Ising models, J. Stat. Phys. 46 (1987) 1159-1194. 
[16] Ledoux M., Log-Sobolev inequality for unbounded spin systems revisited, preprint, 1999.

[17] Liggett T.M., Interacting Particle Systems, Springer Verlag, Berlin, 1985.

[18] Lindvall T., Lectures on the Coupling Method, Wiley, 1992.

[19] Lu S.L., Yau H.T., Spectral gap and logarithmic Sobolev inequality for Kawasaki and Glauber dynamics, Comm. Math. Phys. 156 (1993) 399-433.

[20] Martinelli F., Olivieri E., Approach to equilibrium of Glauber dynamics in the one phase region I: Attractive case, Comm. Math. Phys. 161 (1994) 447-486.

[21] Martinelli F., Olivieri E., Approach to equilibrium of Glauber dynamics in the one phase region II: General case, Comm. Math. Phys. 161 (1994) 487-514.

[22] Stroock D.W., Varadhan S.R.S., Multidimensional Diffusion Processes, Springer Verlag, Berlin, 1979.

[23] Stroock D.W., Zegarlinski B., The equivalence of the logarithmic Sobolev inequality and the Dobrushin-Shlosman mixing condition, Comm. Math. Phys. 144 (1992) 303-323.

[24] Stroock D.W., Zegarlinski B., The logarithmic Sobolev inequality for discrete spin systems on the lattice, Comm. Math. Phys. 149 (1992) 175-193.

[25] Sugiura M., Private communication.

[26] Yoshida N., Sobolev spaces on a Riemannian manifold and their equivalence, J. Math. Kyoto Univ. 33 (1992) 621-654.

[27] Yoshida N., The log-Sobolev inequality for weakly coupled lattice fields, Probab. Theory Related Fields 115 (1999) 1-40.

[28] Yoshida N., Application of log-Sobolev inequality to the stochastic dynamics of unbounded spin systems on the lattice, J. Funct. Anal. 173 (2000) 74-102.

[29] Zegarlinski B., The strong decay to equilibrium for the stochastic dynamics of unbounded spin systems on a lattice, Comm. Math. Phys. 175 (1996) 401-432. 